\title{
An Automatic Classification of Product Review into Given Viewpoints
}

\author{
Yuki Tachizawa, Makoto Oka, and Hirohiko Mori \\ Tokyo City University, 1-28-1 Tamadutumi, Setagaya Tokyo, Japan \\ \{g1281817, moka, hmori\} @tcu.ac.jp
}

\begin{abstract}
Product reviews on the web sites help not only consumers to purchase products but also developers to analyze consumers' needs. Because huge amount of the reviews are presented on the various sites, however, it is a hard task for them to read and to find only the reviews which match their viewpoint that they focus on. Though, to overcome this issue, many researchers in the field of the natural language processing tried to find review sites, classification of reviews according to their viewpoints does not have been succeeded because the corpus for classification must be needed and building it takes a lot of cost. In this paper, we propose a method to build the corpus for each type of products automatically and also propose a method for automatic classification of method of the review. In our method of classification, we focused on the property of review by extending the Tf-Idf. As the classificatoin results contained many errors of classifications in the similar viewpoints, we built the improved method. In this method, we divided the classification process into two-stage. As the result, we could classify reviews by over 80 point.
\end{abstract}

Keywords: human engineering, classification, text mining.

\section{Introduction}

In those days, due to the development of the Internet, we can get much valuable information about many kinds of products and a lot of people can get not only the official information but also the users' subjective reviews of the products. As product reviews contain the feedbacks of using them, it is useful for the consumers in purchasing the items. Such kinds of information are also useful for the developers of the products because, to develop new products, they must grasp the users' feedback. However, it is a hard task to read all reviews and to find the reviews which contain the information that they are interested in. For example, when a reader is interested in the design of the digital camera, the reviews are written from various kinds of viewpoints, such as design, picture quality, portability and so on. Though it should be useful if computer extracts only the reviews which much the users viewpoints he/she focuses on, it is difficult to analyze them by the computers because the reviews are written in free forms sentences. 
Many researchers in the field of the natural language processing tried to classify reviews [1][2][3], few researches has been succeeded so far, because, to do so, the corpuses customized for each products are required and it is very hard to build each corpus for each product manually. a.

In this paper, we, first, propose a method of automatically building of the corpus for each product using some review site as learning data to build the corpus. We also propose two classification of methods of the reviews using the corpus.

\section{Classification}

In this section we describe how to classify review articles in our system. At first, we describe the method of building the corpus automatically. Second, we describe the method of calculating the weight of words. This weight was obtained from the corpus. And it show how much the word has association with each viewpoint. Finally, we describe two method of classifier by using weight that we suggest.

\subsection{Method of Automatically Building of the Corpus}

Corpus is a gallery of the sample text. We picked out characteristics of the viewpoint from this corpus and use characteristics for a classification. As mentioned above, building corpus needs a lot of cost. Here we propose a method to build the corpus automatically.

In those days, rating sites attract somebody's attention. Because of this, reviews that are written in such sites drastically increased. Among these sites, we focus on the site that have templates for reviews. Template means that user dose not write review all in all but write about viewpoints that are made up by the site.

In this experimentation, we adopted "Kakaku.com" as site for corpus. Because lots of people use "Kakaku.com", it has many reviews about various products. And it preliminarily made up some viewpoints for a writer. (Fig.1) Figure 1 is the review about camera. The underline parts are viewpoints that are prepared beforehand. Our system collects reviews to a clue in this every viewpoint.

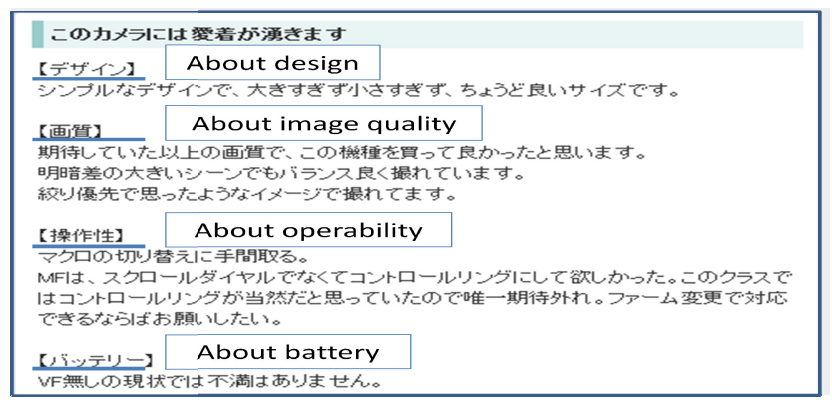

Fig. 1. Review of kakau.com 


\subsection{Weight for Classify}

We use the degree of association with each evaluation of words for classification. Here, the degree of association with each evaluation call weight and describe method of count up the weight of word.

Tf-idf is agenerally used to classify documents. This weight is a statistical measure used to evaluate how important a term is for a document. The importance increases proportionallyby the number of times a term appears in the document but is offset by the frequency of the word in the corpus. We show the expression of Tf-ifd(Eq.1).

$$
\begin{aligned}
& \mathrm{Tf} \mathrm{idf}_{\mathrm{i}, \mathrm{j}}=\mathrm{tf}_{\mathrm{i}, \mathrm{j}} \times \mathrm{idf}_{\mathrm{i}, \mathrm{j}} \\
& \mathrm{tf}_{\mathrm{i}, \mathrm{j}}=\frac{\text { the count of appearance of term } \mathrm{i} \text { in category } \mathrm{j}}{\text { the count of all terms in category } \mathrm{j}} \\
& \operatorname{idf}_{\mathrm{i}, \mathrm{j}}=\log \left(\frac{\text { number of documents }}{\text { the number of documents containing term } \mathrm{i}}\right) \ldots
\end{aligned}
$$

Here, Tf-value is the term frequency in one corpus. Idf-value is the inverse document frequency of term over a collection of among some corpus. Thus, it is the bias of appearance of terms among some documents. Tf-Idf-value is weight which multiplied Tf-value by Idf-value about term.

Table 1 shows the Tf-idf values of the words in each category for the digital singlelens reflex camera. Looking at these results, while the values of the words that appear frequently, such as "design" for the design category, "hold and shooting" for the portability category are too high, the word of "shoulder bag" which should have a high value for the category of "portability" is approximately zero value. Such results caused because Tf-Idf requires many sentences in the document such as articles of newspapers, books, and academic papers, as important terms must be appeared frequently. On the other hand, a product review is not long enough to get the frequency of the important terms. As the result, specific words have too significant effects. It means that certain sentence has been classified in specific evaluation viewpoint when that sentence contains specific word by necessity, and we can find

\begin{tabular}{|c|c|c|c|c|c|c|c|c|}
\hline $\begin{array}{l}\text { category } \\
\text { wrods }\end{array}$ & design & $\begin{array}{l}\text { image } \\
\text { quality }\end{array}$ & operability & battely & portability & function ality & LCD & $\begin{array}{l}\text { feelings of } \\
\text { hold }\end{array}$ \\
\hline design & 28.15 & 0.00 & 0.39 & 0.06 & 0.45 & 0.08 & 0.21 & 1.39 \\
\hline hold & 2.67 & 0.80 & 0.48 & 17.66 & 4.62 & 0.94 & 0.31 & 13.11 \\
\hline shooting & 0.45 & 7.52 & 4.92 & 15.22 & 1.75 & 10.51 & 7.91 & 2.88 \\
\hline do & 18.49 & 21.81 & 20.21 & 27.51 & 14.75 & 18.29 & 21.15 & 17.85 \\
\hline \multicolumn{9}{|l|}{$\ldots$} \\
\hline fatigue & 0.00 & 0.00 & 0.00 & 0.00 & 0.11 & 0.00 & 0.05 & 0.32 \\
\hline sensing & 0.00 & 0.00 & 0.00 & 0.06 & 0.00 & 0.06 & 0.00 & 0.00 \\
\hline shoulder bag & 0.00 & 0.00 & 0.00 & 0.00 & 0.39 & 0.00 & 0.00 & 0.00 \\
\hline error & 0.00 & 0.00 & 0.06 & 0.06 & 0.00 & 0.00 & 0.00 & 0.00 \\
\hline
\end{tabular}
that general Tf-idf does not work well for the classification of review articles.

Table 1. Example of TfIdf-values

We, therefore, extend Tf-Idf to solve this problem and to use to classify reviews. The new weight can be calculated as follows (Eq.2). 


$$
\begin{gathered}
\mathrm{W}_{\mathrm{i}, \mathrm{j}}=\left(\frac{\mathrm{Tfidf}_{\mathrm{i}, \mathrm{j}}}{\sum_{\mathrm{k}=1}^{\mathrm{n}} \mathrm{Tf} \mathrm{idf}_{\mathrm{i}, \mathrm{k}}}\right)^{2} \times \alpha \\
\left\{\begin{array}{l}
\text { if } 0.1>\max \left(\mathrm{Tf} \mathrm{idf}_{\mathrm{i}, 1}, \mathrm{Tf} \mathrm{idf}_{\mathrm{i}, 2}, \ldots, \mathrm{Tf}_{\mathrm{id}, \mathrm{j}}\right) \times 10^{2} \alpha=1 \\
\ldots \\
\text { if } \max \left(\mathrm{Tf} \mathrm{idf}_{\mathrm{i}, 1}, \mathrm{Tf} \mathrm{idf}_{\mathrm{i}, 2}, \ldots, \mathrm{Tf} \mathrm{idf}_{\mathrm{i}, \mathrm{j}}\right) \times 10^{2}>5 \alpha=9 \ldots
\end{array}\right.
\end{gathered}
$$

The first term of expression (1) makes the weight of the bias of appearance of terms strong. The denominator is summarized value of all categories of Tf-idf values about the term. So, the first term represents square of rate of term $\mathrm{i}$ about category $\mathrm{j}$ of Tfidf value. The second term of expression (1), $\alpha$ gives a certain level of weight to term which is low appearance frequency in the extreme. And it adjusts the weight of term which high appearance frequency expressly. (2) is the expression to determine the alpha. Alpha determined depending on the maximal value of Tf-idf among all category. And we configured that it is step function.

Table 2 shows the new weight of the words for each category as same as a table 1(Table 2). Comparing to Table 1, the results has been improved and the extended Tfidf can be said to work well for the classification of the review articles.

Table 2. Example of new weight

\begin{tabular}{|c|c|c|c|c|c|c|c|c|}
\hline $\begin{array}{l}\text { category } \\
\text { wrods }\end{array}$ & design & $\begin{array}{l}\text { image } \\
\text { quality } \\
\end{array}$ & operability & battely & portability & functionality & LCD & $\begin{array}{l}\text { feelings of } \\
\text { hold }\end{array}$ \\
\hline design & 8.24 & 0.00 & 0.12 & 0.02 & 0.13 & 0.02 & 0.06 & 0.41 \\
\hline hold & 0.59 & 0.18 & 0.11 & 3.91 & 1.02 & 0.21 & 0.07 & 2.91 \\
\hline shooting & 0.08 & 1.32 & 0.87 & 2.68 & 0.31 & 1.85 & 1.39 & 0.51 \\
\hline do & 1.04 & 1.23 & 1.14 & 1.55 & 0.83 & 1.03 & 1.19 & 1.00 \\
\hline \multicolumn{9}{|l|}{$\ldots$} \\
\hline fatigue & 0.00 & 0.00 & 0.00 & 0.00 & 0.68 & 0.00 & 0.33 & 1.99 \\
\hline sensing & 0.00 & 0.00 & 0.00 & 0.53 & 0.00 & 0.47 & 0.00 & 0.00 \\
\hline shoulder bag & 0.00 & 0.00 & 0.00 & 0.00 & 3.00 & 0.00 & 0.00 & 0.00 \\
\hline error & 0.00 & 0.00 & 0.47 & 0.53 & 0.00 & 0.00 & 0.00 & 0.00 \\
\hline
\end{tabular}

When compared with Table 1, weight of design fall and weight of shoulder bag rise. The problem of Tf-Idf is solved.

\subsection{Morphological Analysis}

Though there is a space between words in English, there is no space in Japanese language. So, in processing natural language in Japanese, it is required to divide a sentence into each word. Chasen is a Japanese morphological analysis software based on support vector machines. It cut up Japanese language that is no separated to morpheme unit and we can obtain the information about the word class, its antetype and so on (Figure 2.) The first line is an input to Chasen in Japanese, which means "I am alumnus of Tokyo City University". From the second line, the first rows show information of divided term. The second and third rows shows the antetype of the term presented in first row, and the forth rows show the word class.

In this method, we utilize the antetype of the noun, verb, adjective and adjective to classify the documents. 


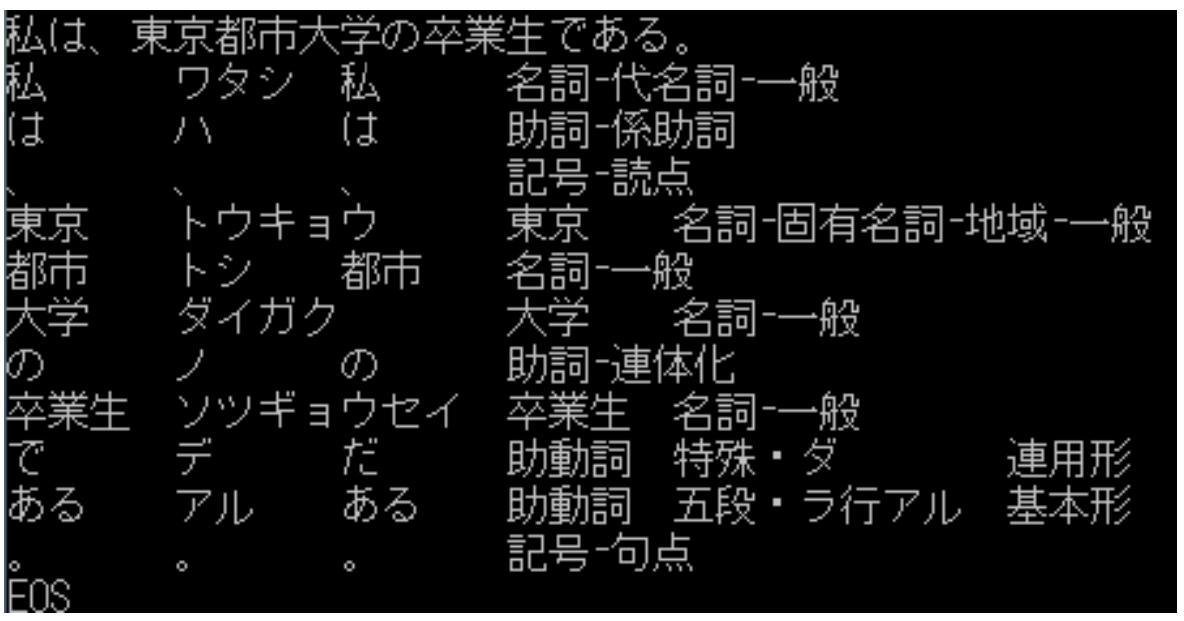

Fig. 2. Output of Japanese morphological analysis "Chasen"

\subsection{Classification}

Here, we explain how we classify with the weight. When we presuppose that each category express $\left\{\mathrm{C}_{1}, \mathrm{C}_{2}, \mathrm{C}_{3}, \ldots, \mathrm{C}_{\mathrm{i}}\right\}$ and sentence express $\left\{\mathrm{S}_{1}, \mathrm{~S}_{2}, \mathrm{~S}_{3}, \ldots, \mathrm{S}_{\mathrm{j}}\right\}$ and words are contained sentence express $\left\{\mathrm{W}_{1}, \mathrm{~W}_{2}, \mathrm{~W}_{3}, \ldots, \mathrm{W}_{\mathrm{k}}\right\}$ and the weight which the word $\mathrm{k}$ has about category i express $V_{i, n}$, the classification result $\widehat{C}$ get decided by following expression.( Eq.3)

$$
\begin{aligned}
f(A, j) & =V_{A, 1}+V_{A, 2}+V_{A, 3}+\cdots+V_{A, n} \\
& =\sum_{\mathrm{k}=1}^{n} V_{A, k} \\
\hat{C} & =\underset{C_{i}}{\arg \max } f(i, j) \\
& =\underset{C_{i}}{\arg \max } \sum_{l=1}^{n} V_{A, l} \cdots
\end{aligned}
$$

$f(A, j)$ is total of weight of category A about sentence $j$.

This expression computes total of each category's weight of words which are included in object sentence and adopts the category that is the highest total weight as result.

\section{Experiment}

To evaluate our system, we used the review articles of the digital camera in "kakaku.com" to build the corpus and adopted our system to ones in "amazon.com." We made 50 evaluation data about the category of "design", 50 about "image quality", 50 about "operability", 26 about "battery", 50 about "portability", 50 about "functionality," and 50 about "LCD". 


\subsection{Result}

Table 3 indicates the result of classification by our method. As F-values of most of viewpoints are over 80, it can be said that the weight in our approach is good for classifications of the reviews. Here, "image quality" and "functionally" are fewer than 70. When we watch a careful result (Table 4), there were many errors for the classification between the "image quality" and the "functionally", between "portability" and "hold", and between "image quality" and "LCD".

Table 3. Accuracy of our method of classification

\begin{tabular}{|l|r|r|r|}
\hline & Presision(\%) & Recall(\%) & F-value (\%) \\
\hline design & 84 & 88 & 85 \\
\hline image quality & 71 & 66 & 68 \\
\hline operability & 86 & 88 & 86 \\
\hline battely & 92 & 100 & 95 \\
\hline portability & 72 & 96 & 82 \\
\hline functionality & 85 & 58 & 68 \\
\hline LCD & 73 & 92 & 81 \\
\hline feelings of hold & 88 & 64 & 74 \\
\hline totality & 80 & 80 & 80 \\
\hline
\end{tabular}

Table 4. A classification result of our method of classification

\begin{tabular}{|l|r|r|r|r|r|r|r|r|r|}
\hline & design & image & opera $\cdots$ & battely & porta $\cdots$ & func $\cdots$ & LCD & hold & answer \\
\hline design & 44 & 1 & 0 & 0 & 2 & 0 & 0 & 3 & 50 \\
\hline image & 2 & 33 & 0 & 1 & 0 & 0 & 14 & 0 & 50 \\
\hline operability & 2 & 0 & 44 & 0 & 3 & 1 & 0 & 0 & 50 \\
\hline battely & 0 & 0 & 0 & 26 & 0 & 0 & 0 & 0 & 26 \\
\hline portability & 0 & 0 & 0 & 0 & 48 & 1 & 0 & 1 & 50 \\
\hline functionality & 1 & 12 & 6 & 0 & 0 & 29 & 2 & 0 & 50 \\
\hline LCD & 0 & 0 & 1 & 0 & 0 & 3 & 46 & 0 & 50 \\
\hline hold & 3 & 0 & 0 & 1 & 13 & 0 & 1 & 32 & 50 \\
\hline output & 52 & 46 & 51 & 28 & 66 & 34 & 63 & 36 & \\
\hline
\end{tabular}

\subsection{Consideration}

Though we could obtain the good results by proposed our method as a whole, there are many errors between some of the viewpoints.

Analizing the results in detail, the same terms which characterize the categories are contained over two categories. For example, the term of "the image quality" is contained in both categories of "image quality" and "LCD", because the reviewers sometimes evaluated "LCD" from its quality. In this way, the appearance frequency of the word come close in each other.

Analizing results also show that some errors were caused by the ambigious meaning of terms. As, for example, we a Japanese word "Motsu" that have both meanings of "carry" and "have", the term "Motsu" must have large weight values in both categories of "portability" and "feeling of hold". However, in our method of classification, the weight of "Motsu" only in the category "feeling of hold" was large while the one in the category "portability" was small.

Here, note that the terms such as "Motsu" are useful to distinguish among a combined category of "feeling of hold" and "portability" and another categories. 


\section{Improved Classfication}

In the above consideration, we mentioned the current problems of the system. To drawback them, we improved the way of the classification.

\subsection{Improved Method}

When we try to classify something, we sometimes divide them into some big categories roughly before classifying them into the detail categories. Our improved system mimics this way and adopts two-stage classification, which is as follows:

1. The system makes some groups of the categories. This process is done be the cluster analysis using the frequency of each term.

2. The importance weights of appeared terms for each big category are calculated.

3. Using the importance weights, the review articles are classified in to the big groups.

4. The importance weights of appeared terms for the small categories are calculated, considering each big group as one entire articles.

5. The terms which have the close importance weights over the multiple categories are eliminated.

6. The classification into the small categories is done in the same manner as above.

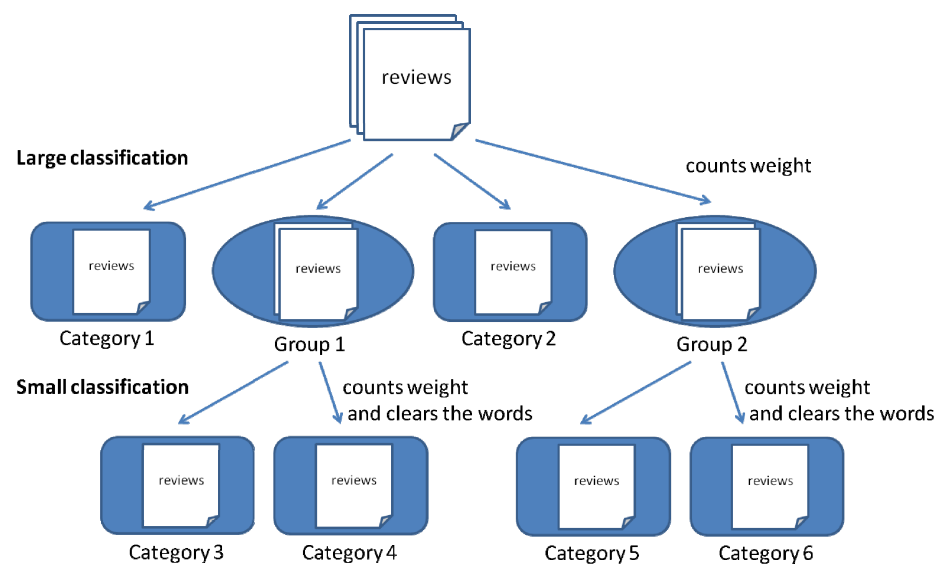

Fig. 3. Flowing of the method of improved classification

As a result of step 1, "image quality" and "LCD" ,"Feeling of hold" and "Portability", "operability" and "functionally" were got together. Most of word that were problem about mistake in near evaluation viewpoints in hindsight of step 5. 


\section{$5 \quad$ Experiment II}

To compare the results between the experiment I and II, we used the same data.

\section{$5.1 \quad$ Result}

The table 5 shows that, comparing with the experiment I, the whole results are improved and, especially, the F-values of the categories whose results were not obtained the good results, such as the categories of "image quality", "portability", "functionality", "LCD", and "feelings of hold" are raised. It can be said, therefore, the two-stage classification drawback the problem of the first manner of the classification.

Table 5. Accuracy of improved classification

\begin{tabular}{|l|r|r|r|}
\hline & Presision(\%) & Recall(\%) & F-value(\%) \\
\hline design & 84 & 88 & 85 \\
\hline image quality & 72 & 74 & 72 \\
\hline operability & 89 & 84 & 86 \\
\hline battely & 92 & 100 & 95 \\
\hline portability & 82 & 92 & 86 \\
\hline functionality & 81 & 62 & 70 \\
\hline LCD & 79 & 92 & 85 \\
\hline feelings of hold & 84 & 78 & 80 \\
\hline totality & 83 & 83 & 83 \\
\hline
\end{tabular}

Table 6. A classification result of improved classfication

\begin{tabular}{|l|r|r|r|r|r|r|r|r|r|}
\hline & design & image & opera $\cdots$ & battely & porta $\cdots$ & func $\cdots$ & LCD & hold & an swer \\
\hline design & 44 & 1 & 0 & 0 & 1 & 0 & 0 & 4 & 50 \\
\hline image & 2 & 37 & 0 & 1 & 0 & 0 & 10 & 0 & 50 \\
\hline operability & 2 & 0 & 42 & 0 & 3 & 3 & 0 & 0 & 50 \\
\hline battely & 0 & 0 & 0 & 26 & 0 & 0 & 0 & 0 & 26 \\
\hline portability & 0 & 0 & 0 & 0 & 46 & 1 & 0 & 3 & 50 \\
\hline functionali & 1 & 13 & 4 & 0 & 0 & 31 & 1 & 0 & 50 \\
\hline LCD & 0 & 0 & 1 & 0 & 0 & 3 & 46 & 0 & 50 \\
\hline hold & 3 & 0 & 0 & 1 & 6 & 0 & 1 & 39 & 50 \\
\hline output & 52 & 51 & 47 & 28 & 56 & 38 & 58 & 46 & 376 \\
\hline
\end{tabular}

\subsection{Consideration}

By the two-stage classification, we could improve the precision of classification between the similar viewpoints. Eliminating the terms which appear frequently in multiple categories, however, causes another problem and, eventually causes another type of the classification error. In this process, too many terms were eliminated including some important terms. For example, the review sentence "The feeling of holding this camera is comfortable" was not classified into the "Hold" category but into the "Portability", because the term "hold" and "comfortable" were removed and only the term "feeling" remained. To solve this problem, another parameter in addition to the frequency should be considered in removing redundant terms. 


\section{Consideration the of Two Methods}

We found the new calcuration manner of the importance weights and the two-stage classification succeeded to classify of review articles. This manner should be able to adopt to the classification of another small documents. But some problems is still left to solve.

One is how to deal with compound nouns. In our method, a compound noun are treated as a aggregate of words and theie weights are calculated independently. For example, when the words "Vari-angle LCD monitor function" are contained in the article, it is classified into the "LCD" category because the weight of term "LCD“" have a large value for the "LCD" category, while the article should be classified into the category "function." If we can identify "Vari-angle LC monitor function" as a one compound norn and give one weight value to it, this article would be classified into the category "function." To solve this problem, we need to develop some manners to identify compund nouns.

The second is that, when an article includes a word which has extreme high weight for one category, it is classified into the category because of too strong affect of the word. To refine the calculation method of weight may solve this problem .

\section{Conclusion}

In this paper, we propose a method to build the corpus and also propose a method for automatic classification of the review articles. Our system works out over $80 \%$ accuracy in classification.

In our method we use only the frequency of the appeared word to classify reviews. But, only with values, it should be difficult to get the result of the classification more precisely. Now, we are considering to utilize sentence structures. Especially, using the grammatical modification relations would be contribute to more precious.

\section{$8 \quad$ Future Works}

In our method we use only he appearance frequency information of the word. But, only with this information, it is difficult to raise classification precision. We assume that we use information of modification relation to raise classification precision.

About the compound noun, we would identify it and give heaviness by different methods of words.

\section{References}

1. Kaji, N., Kitsuregawa, Y.: The automatic collection of evaluation sentences from an HTML document galaxy. Natural Language Processing Association (2004)

2. Suzuki, M.: The automatic classification of the text with difference of the word frequency between categories. Journal of Japan Industrial Management Association (2008) 